"The submitted manuscript has been authored by a contractor of the U.S. Government under contract No. DE-AC05-96OR22464. Accordingly, the U.S. Government retains a nonexclusive royalty-free license to publish or reproduce the published form of this contribution, or allow others to do so, for U.S. Government purposes."

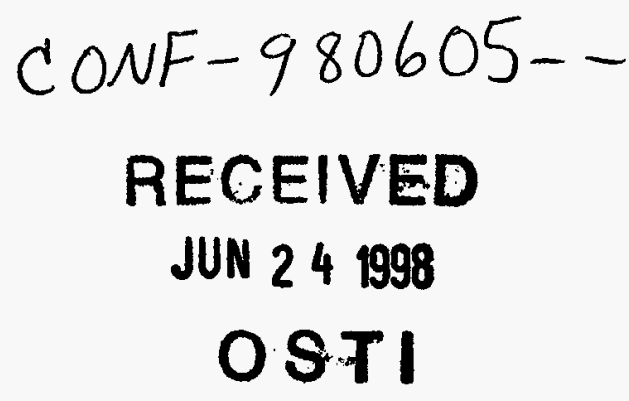

\title{
Tritium Concentration Measurements in the JET Divertor by Optical Spectroscopy of a Penning Discharge*
}

\author{
by
}

\author{
D. L. Hillis
}

Oak Ridge National Laboratory

Oak Ridge, Tennessee, USA

P. D. Morgan, J. K. Ehrenberg, M. Groth, M. F. Stamp, and $M$. von Hellermann

JET Joint Undertaking

Abingdon, Oxfordshire, U. K.

\section{Kumar}

Institute for Plasma Research

Bhat, Gandhinagar 382-428, India

Presented at the

12th Topical Conference on High-Temperature Plasma Diagnostics

Princeton, New Jersey

June 7-11, 1998

To be published in

Review of Scientific Instruments

OASTAIBUTION OF THIS DOCUMENT is UNLIMITED ph MASTER

*Research sponsored in part by the U.S. Department of Energy, Office of Fusion Energy Sciences, under contract DE-AC05-960R22464 with Lockheed Martin Energy Research Corporation. 


\section{DISCLAIMER}

This report was prepared as an account of work sponsored by an agency of the United States Government. Neither the United States Government nor any agency thereof, nor any of their employees, makes any warranty, express or implied, or assumes any legal liability or responsibility for the accuracy, completeness, or usefulness of any information, apparatus, product, or process disclosed, or represents that its use would not infringe privately owned rights. Reference herein to any specific commercial product, process, or service by trade name, trademark, manufacturer, or otherwise does not necessarily constitute or imply its endorsement, recommendation, or favoring by the United States Government or any agency thereof. The views and opinions of authors expressed herein do not necessarily state or reflect those of the United States Government or any agency thereof. 


\title{
Tritium concentration measurements in the JET divertor by optical spectroscopy of a Penning discharge*
}

\author{
D.L. Hillis ${ }^{a}$, P. D. Morgan ${ }^{\ddagger}$, J.K. Ehrenberg ${ }^{\ddagger}$, M. Groth ${ }^{\ddagger}$, V. Kumar ${ }^{\dagger}$, \\ M.F. Stamp $\ddagger$, and $\ddagger$, M. von Hellermann $\ddagger$, \\ Oak Ridge National Laboratory, Oak Ridge, Tennessee 37831 USA \\ ${ }^{\ddagger} J E T$ Joint Undertaking, Abingdon, Oxfordshire, UK \\ ${ }^{\dagger}$ Institute for Plasma Research, Bhat, Gandhinagar 382-428, India
}

\section{ABSTRACT}

Obtaining precision measurements of the relative concentrations of hydrogen, deuterium, tritium, and helium in the divertor of a tokamak are an important task for nuclear fusion research. Control of the deuterium-tritium isotopic ratio while limiting the helium ash content in a fusion plasma are key factors for optimizing the fuel burn in a fusion reactor, like the International Tokamak Experimental Reactor (ITER). A diagnostic technique has been developed to measure the deuterium-tritium isotopic ratio in the divertor of the Joint European Torus (JET) with a species-selective Penning vacuum gauge. The Penning discharge provides a source of electrons to excite the neutral hydrogen isotopes in the pumping duct. Subsequently, the visible light from the hydrogen isotopes is collected in an optical fiber bundle, transferred away from the tokamak into a low radiation background area, and analyzed in a high resolution Czerny-Turner spectrometer, which is equipped with a fast charge coupled device (CCD) camera for optical detection. The intensity of the observed line emission ( $D_{\alpha}-6561.03 \AA$; and $\left.T_{\alpha}-6560.44 \AA\right)$ is directly proportional to the partial pressure of each gas found in the divertor. The line intensity of each isotope is calibrated as a function of pressure. The ratio of the line intensities thus provides a direct measurement of the deuterium-tritium isotopic ratio. The lower limit for the determination of the deuterium-tritium isotopic ratio is about $0.5 \%$. The applicable pressure range for this system is from $10^{-5} \mathrm{mbar}$ to a few times $10^{-3} \mathrm{mbar}$.

a) Electronic mail: HillisDL@ornl.gov 


\section{INTRODUCTION}

Controlling the deuterium-tritium isotopic ratio while limiting the helium ash content are key elements to creating and optimizing the fuel burn in future fusion reactors, i.e., ITER. Preferential isotope enrichment is also an important indicator of particle confinement processes. For these reasons, the measurement of the relative and absolute concentrations of hydrogen, deuterium, tritium, and helium is of considerable interest in the JET deuterium-tritium experiments and for minority radio frequency (RF) heating experiments where the minority isotope species (usually hydrogen or helium) must be accurately known. On the Tokamak Fusion Test Reactor (TFTR), a diagnostic system ${ }^{1}$ to measure the deuterium-tritium concentration utilized a Fabry Perot interferometer to scan the visible Balmer-alpha line emission from deuterium and tritium, which are excited in the plasma scrape off layer. Due to the electron temperature (20-100 eV) of the scrape off layer plasma and fast reflected particles, the deuterium and tritium spectral lines are Doppler broadened. Since the visible hydrogen isotope lines are very close together, they tend to blend together and become unresolved, thereby severely limiting the determination of the deuterium-tritium concentration. Here, we report on the application of a species-selective Penning discharge ionization gauge to measure, simultaneously, the Balmeralpha emission from the hydrogen isotopes (hydrogen, deuterium, and tritium) in the divertor chamber of JET via a spectroscopic technique, as well as the concentration of other impurity gases in the divertor, such as helium. The conceptual design of this diagnostic was first reported in Ref. 2.

The Penning gauge produces a cold plasma discharge $(-5 \mathrm{eV})$; thereby producing very narrow spectral lines for $H_{\alpha}, D_{\alpha}$, and $T_{\alpha}$, which are easily resolved. The diagnostic system described here successfully operated throughout the JET deuterium-tritium experiments, which were performed in 1997. Several of the other tritium measurements on JET are described in Ref. 3. 


\section{EXPERIMENTAL ARRANGEMENT}

The Penning gauge system is installed at the end of a 2.5 meter vacuum tube which is connected to a lower sub-divertor port of JET and located near the cryopumping plenum of the JET MkII divertor (see Fig. 1). The location of the Penning gauge is in a region where the magnetic fields from JET are less than 100 gauss, to avoid interaction with the Penning gauge operation. The deuterium, tritium, and other impurity species found in the divertor travel along the vacuum duct to the Penning gauge and are ionized by the Penning discharge. The Penning gauge is a commercial vacuum gauge: Alcatel model CF 2 P. The visible light emitted by the gas discharge in the Penning gauge is viewed spectroscopically via a heated one-millimeter quartz fiber (to prevent browning during deuterium-tritium operation) and transferred 80 meters away from the tokamak to a low-radiation area, where the spectrometer system is located. The first 25 meters of the quartz fiber, which are within the JET biological radiation shield, were protected by an aluminum jacket and enclosed in a metal insulated tube which is heated to $250^{\circ} \mathrm{C}$. During deuterium-tritium operation the fiber transmission can be substantially reduced from browning of the quartz fibers (production of color centers) due to the influence of neutron and high radiation interactions with the fibers. This effect is avoided by heating the fibers to $250^{\circ} \mathrm{C}$ and thermally annealing out the color centers as soon as they form. ${ }^{4.5}$ The fiber transmission and possible luminescence of the Penning gauge fibers was monitored throughout the deuterium-tritium experiments and neither was found to have a significant effect.

The original concept of using a Penning discharge ionization gauge, combined with spectroscopic views of the discharge, was developed to provide helium concentration measurements in the pumping ducts of the TEXTOR ALT-II pump limiter and was described in Refs. 6 and 7. Unlike the application for helium partial pressure measurements, the measurement of the deuterium and tritium partial pressures (or concentration) necessitates the use of a high resolution spectrometer (1.0 meter Czerny-Turner) with an 1800 lines $/ \mathrm{mm}$ grating. This spectrometer arrangement permits the simultaneous separation of the visible Balmer transitions 
for the various hydrogen isotopes $\left(H_{\alpha}-6562.79 \AA\right.$; $D_{\alpha}-6561.03 \AA$; and $\left.T_{\alpha}-6560.44 \AA\right)$ using multi-Gaussian fitting techniques. Due to the low temperature of the Penning discharge, the width of the hydrogen spectral lines is very narrow (determined mainly by the instrument function of the spectrometer). This makes it possible to easily separate the line intensities of the three hydrogen isotopes. The intensity of the corresponding lines is a linear function of the pressure in the desired range of $10^{-5} \mathrm{mbar}$ to a few times $10^{-3} \mathrm{mbar}$ and the line intensity is easily related to the partial pressures of deuterium and tritium. The spectrometer is equipped with a state-of-the-art CCD-based detector, which enables rapid readout of the visible spectra. ${ }^{8}$ The minimum integration time of the CCD camera detector system is about $10 \mathrm{~ms}$, but the time response of the diagnostic is limited by the conductance between the divertor and the Penning gauge $(\sim 150 \mathrm{~ms})$. The spectrometer and CCD detection system has a dispersion of 0.107 Angstrom/pixel. Measurements in the laboratory and in situ measurements using $\mathrm{H}_{2}, \mathrm{D}_{2}$, and $\mathrm{T}_{2}$ mixtures have been used to characterize the system. Figure 2 shows the typical resolution for the diagnostic system with: (a) a $10 \%$ deuterium $90 \%$ hydrogen calibration mixture and (b) an actual plasma discharge on JET with an isotope mix of $55 \%$ tritium $43.5 \%$ deuterium with a residual background of about $1.5 \%$ hydrogen, which is always present in JET. On-line calibration of the diagnostic on JET for the light intensity versus deuterium or tritium pressure in the divertor is obtained from the gas injection and mixing system shown in Fig. 1 or by injecting deuteriumtritium gas mixtures directly into JET without plasma present.

\section{APPLICATION OF THE PENNING GAUGE}

The Penning gauge is typically used for measuring vacuum pressures in the range of $10^{-6}$ mbar to $10^{-3} \mathrm{mbar}$. In this range the Penning current is a linear function of the pressure and is calibrated and used for a pressure indication. It is also found that the light intensity emitted from the discharge is also proportional to the pressure in the Penning gauge. Figure 3(a) shows the relative intensity of the $D_{\alpha}(6561.03 \AA)$ light as a function of pressure. The intensity of the line is a linear function of the pressure in the desired range of $10^{-5} \mathrm{mbar}$ to $10^{-3} \mathrm{mbar}$. At higher 
pressures the intensity saturates (no longer in the Penning regime) and at lower pressures the Penning discharge extinguishes. The pressure range of the system can be extended to higher pressures ( $\left.10^{-1} \mathrm{mbar}\right)$ by differentially pumping the Penning gauge system and using the variable iris aperture (see Fig. 1). The data of Fig. 2(a) is for a pressure of $0.6 \times 10^{-3} \mathrm{mbar}$. The pixel location of the $H_{\alpha}$ and $D_{\alpha}$ lines is slightly shifted in the two figures. This is due to a repositioning of the lines on the $\mathrm{CCD}$ detector and not to any instrumental drifts.

The data of Fig. 2 are well described by a multi-Gaussian distribution. Each of the visible lines of tritium, deuterium, and hydrogen is described by two Gaussian distributions. The first is a narrow Gaussian that is determined by the instrument function of the spectrometer (FWHM $0.3 \AA$ ) and the second is a wider component (FWHM $\sim 5 \mathrm{eV}$ ), which is the Franck-Condon distribution (see Fig. 2a). Figure 3(b) illustrates how the concentration of one of the hydrogenic isotopes can be determined from the light intensity measured with the Penning gauge. In Fig. 3(b) the relative intensity of $D_{\alpha}$ to the total $D_{\alpha}+H_{\alpha}$ intensity (including both the broad and narrow spectral components in the integration) as measured with the Penning gauge system is plotted as a function of the known concentration of $D_{2}$ in a variety of calibration mixtures of hydrogen and deuterium. Clearly, the deuterium concentration of the calibration mixture can be accurately determined directly from the ratio of $D_{\alpha} /\left(D_{\alpha}+H_{\alpha}\right)$ intensity, which is excited by the Penning gauge. The resulting linear dependence makes this a simple technique for determining the hydrogen, deuterium, or tritium concentrations in fusion plasma experiments. The calculation includes the measured response of the Penning gauge including the Franck-Condon distributions for each isotope. The hydrogen isotopes are easily separated and can be analyzed with a multiGaussian fitting algorithm to deduce the deuterium-tritium concentration from the intensity ratios measured for each visible line.

Figure 4 shows a deuterium-tritium discharge on JET which is injected with a tritium gas puff and heated by $6 \mathrm{MW}$ of RF power. The tritium concentration is measured versus time with the Penning gauge every $250 \mathrm{~ms}$ throughout the discharge. For this JET discharge the tritium concentration is found to rise to about $50 \%$, following the tritium gas injection. In addition to 
the tritium concentration the Penning gauge also measures the pressure in the JET divertor, which is compared with an ionization (ASDEX) gauge located in the divertor pumping plenum. The agreement between the two pressure measurements is excellent. The time resolution of the Penning gauge and light detection system is of the order of a few milliseconds, but the time response is limited by the conductance of the vacuum ducts to the Penning gauge $(\sim 150 \mathrm{~ms})$ as can be seen in comparison with the faster time response of the ASDEX ionization gauge pressure trace.

The species-selective Penning gauge diagnostic was used throughout the JET deuteriumtritium experimental campaign of 1997. Figure 5 shows the tritium concentration measured with the Penning gauge at a time of $56 \mathrm{~s}$ into the discharge for over $200 \mathrm{JET}$ shots with tritium concentrations ranging from $0.3 \%$ to $98 \%$. The Penning gauge measurement is also compared with the tritium concentration measured via a divertor spectroscopic measurement and a core neutron measurement. Excellent agreement is found between the various diagnostic techniques for measuring the tritium concentration. Following JET shot 41760 no more tritium was introduced into the tokamak and various techniques were investigated to speed up liberating tritium from the vessel walls, so the tritium concentration continues to decrease for subsequent discharges. Near JET shots 41790 and 41820 the tritium concentration increases due to high power RF heating, which heats up the vessel walls and mobilizes tritium which was previously trapped in the walls, thereby increasing the tritium concentration for a few discharges.

\section{SUMMARY AND CONCLUSIONS}

A diagnostic technique has been developed and implemented to measure the deuteriumtritium isotopic ratio in the divertor of a tokamak with a Penning vacuum gauge. The Penning discharge excites the neutral deuterium and tritium in the pumping duct. Subsequently, the visible light from the hydrogen isotopes is collected in a heated optical fiber bundle, transferred away from the tokamak into a low radiation background area, and detected in a high-resolution Czerny-Turner spectrometer, which is equipped with a CCD detector system. The spectrometer 
system has a resolution of 0.107 Angstrom/pixel which is adequate to separate the $D_{\alpha}$ and $T_{\alpha}$ transitions. The intensity of the two lines is calibrated as a function of the partial pressures of deuterium and tritium. The ratio of the pressures provides a direct measure of the tritium concentration in the divertor pumping plenum. This technique is applicable for the pressure range from $10^{-5} \mathrm{mbar}$ to a few times $10^{-3} \mathrm{mbar}$. The time resolution of the Penning gauge and light detection system is of the order of a few milliseconds, but generally the time response is limited by the conductance of the vacuum ducts to the Penning gauge ( $150 \mathrm{~ms}$ for the JET case). The lower limit for the determination of the tritium concentrations is about $0.5 \%$. The Penning gauge diagnostic provided fast, accurate, and reliable measurements of the tritium concentration for every JET discharge throughout the 1997 deuterium-tritium experimental campaign, as well as, during the tritium clean-up phase once the tritium experiments were completed. The Penning gauge will continue to be utilized on JET by extending these measurements to include concentration measurements of impurity species, such as helium, nitrogen, neon, and argon, which will be used with the new gas box divertor design.

\section{ACKNOWLEDGMENTS}

The authors are grateful to O.N. Jarvis for providing tritium concentration measurements from the neutron measurements. This research is sponsored in part by the Office of Fusion Energy, U. S. Department of Energy, under Contract DE-AC05-96OR22464 with Lockheed Martin Energy Research Corporation. 


\section{REFERENCES}

'C.H. Skinner, et al., Princeton Plasma Physics Laboratory Report, PPPL-2878, February 1993.

${ }^{2}$ D.L. Hillis, et al., Fusion Engineering and Design 34-35, $347 \quad$ (1997).

${ }^{3}$ P. Andrew, et al., Nuclear Fusion, 33, 1 (1993).

${ }^{4}$ W. Tighe et al., Rev. Sci. Instrum. 66, 907 (1995).

${ }^{5}$ A.T. Ramsey et al., Rev. Sci. Instrum. 68, 632 (1997).

${ }^{6}$ K.H. Finken, K. H. Dippel, and A. Hardtke, Rev. Sci. Instrum. 63, 1 (1992).

${ }^{7}$ G. Mank, et al., Proc. 1994 Conf. Contr. Fusion and Plasma Physics (Montpellier: European Physical Society) Vol. 18B Part 1, p. 130 (1994).

${ }^{8}$ M.G. von Hellermann and H.P. Summers, Atomic and Plasma-Material Interaction Processes in Controlled Thermonuclear Fusion, Ed. Janev, Elsevier Science Publishers, 135 (1993). 


\section{FIGURE CAPTIONS}

Fig. 1. Penning gauge diagnostic system for the measurement of the tritium concentration in the divertor of JET.

Fig. 2. Deuterium and hydrogen Balmer alpha spectra obtained from the species-selective Penning gauge diagnostic for (a) a 10\% deuterium and $90 \%$ hydrogen calibration mixture. The data are the open circles and the lines are a multi-Gaussian fit to the data (see text); (b) tritium, deuterium, and hydrogen Balmer alpha spectra obtained from an actual JET discharge with tritium (55\%) and deuterium (43.5\%) gas fueling with a residual hydrogen background (1.5\%).

Fig. 3. (a) Relative Intensity of the deuterium Balmer alpha (narrow + broad components) as a function of the total deuterium pressure. (b) The ratio of intensities $D_{\alpha}\left(D_{\alpha}+H_{\alpha}\right)$ versus the known concentration of $D_{2}$ in the $\mathrm{H}_{2}+\mathrm{D}_{2}$ calibration mixture. The $\mathrm{D}_{2}$ concentration of the calibration mixture can be determined directly from the ratio of the Balmer alpha line intensities.

Fig. 4. A deuterium-tritium discharge on JET which is injected with a tritium gas puff and heated by $6 \mathrm{MW}$ of RF heating power. The tritium concentration is measured versus time with the Penning gauge throughout the discharge. The subdivertor total pressure is measured via the Penning gauge and is also compared with an ionization (ASDEX) gauge located in the divertor pumping plenum.

Fig. 5. Tritium concentration, $\mathrm{T} /(\mathrm{H}+\mathrm{D}+\mathrm{T})$, measured via Penning gauge throughout the JET deuterium-tritium experimental campaign at time of $56 \mathrm{~s}$ for a sequence of JET deuterium-tritium shots. The tritium concentration from the Penning gauge is compared with tritium concentrations measured with divertor spectroscopy and neutron diagnostics. 


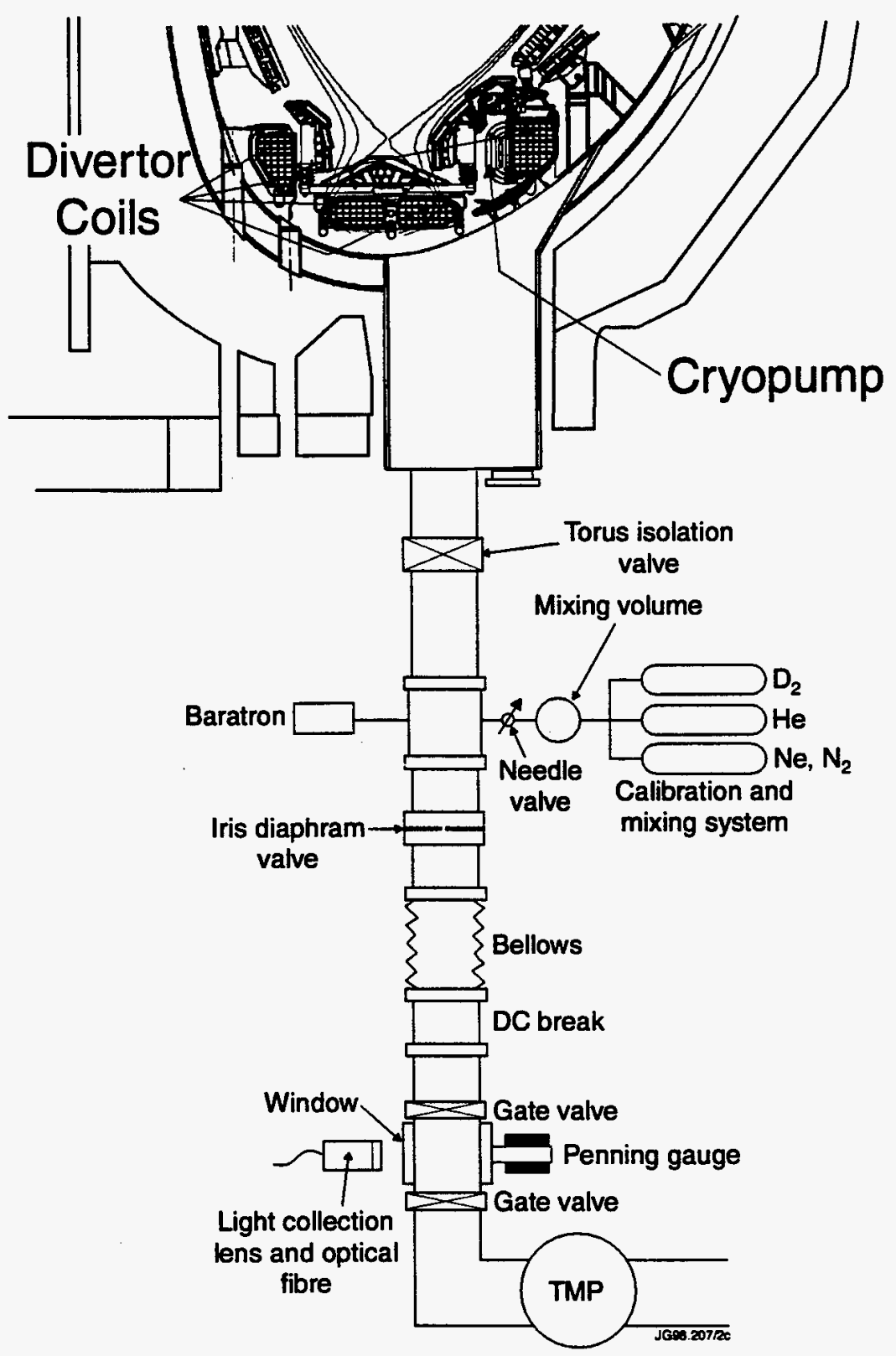

Fig. 1 


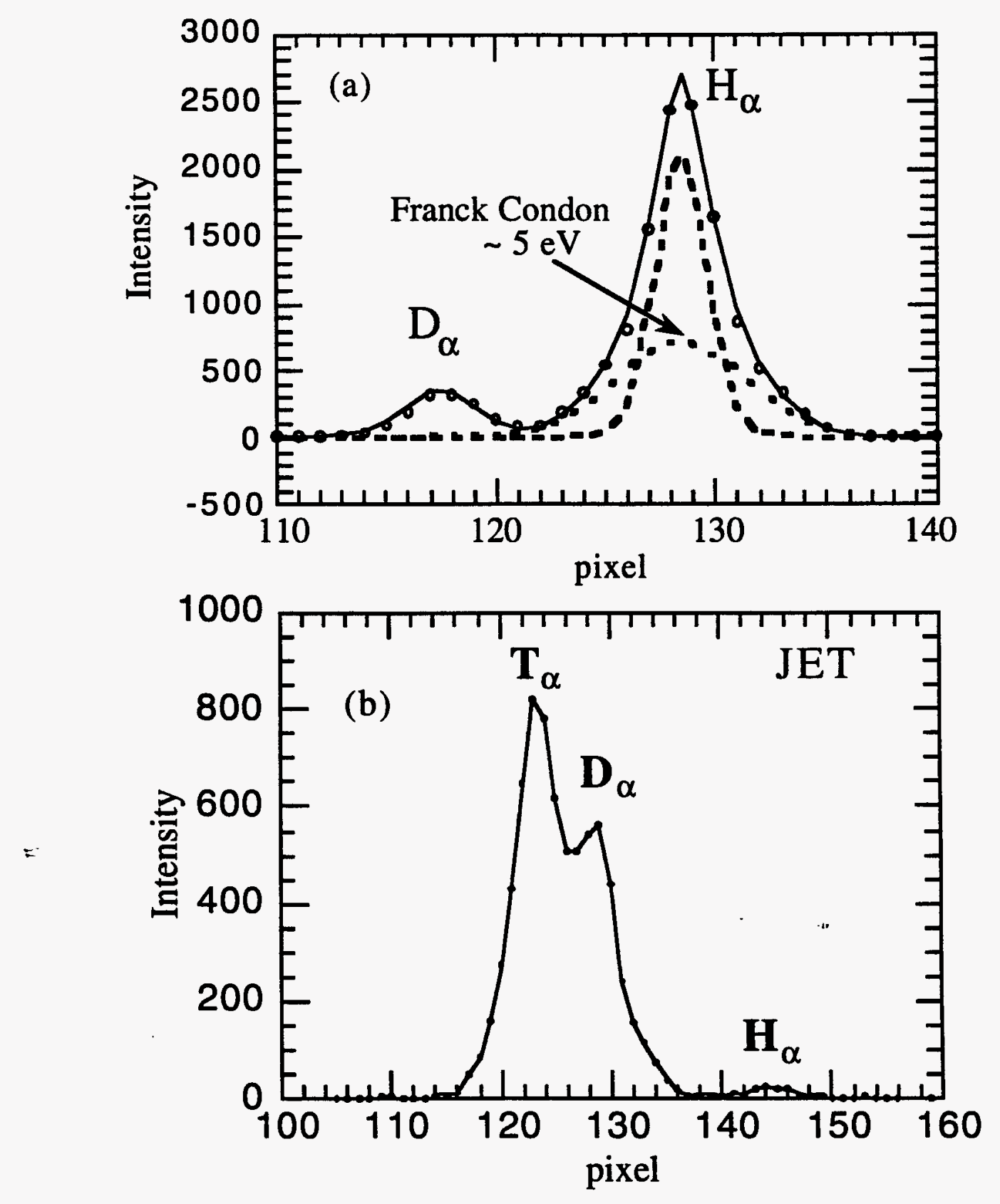

Fig. 2 


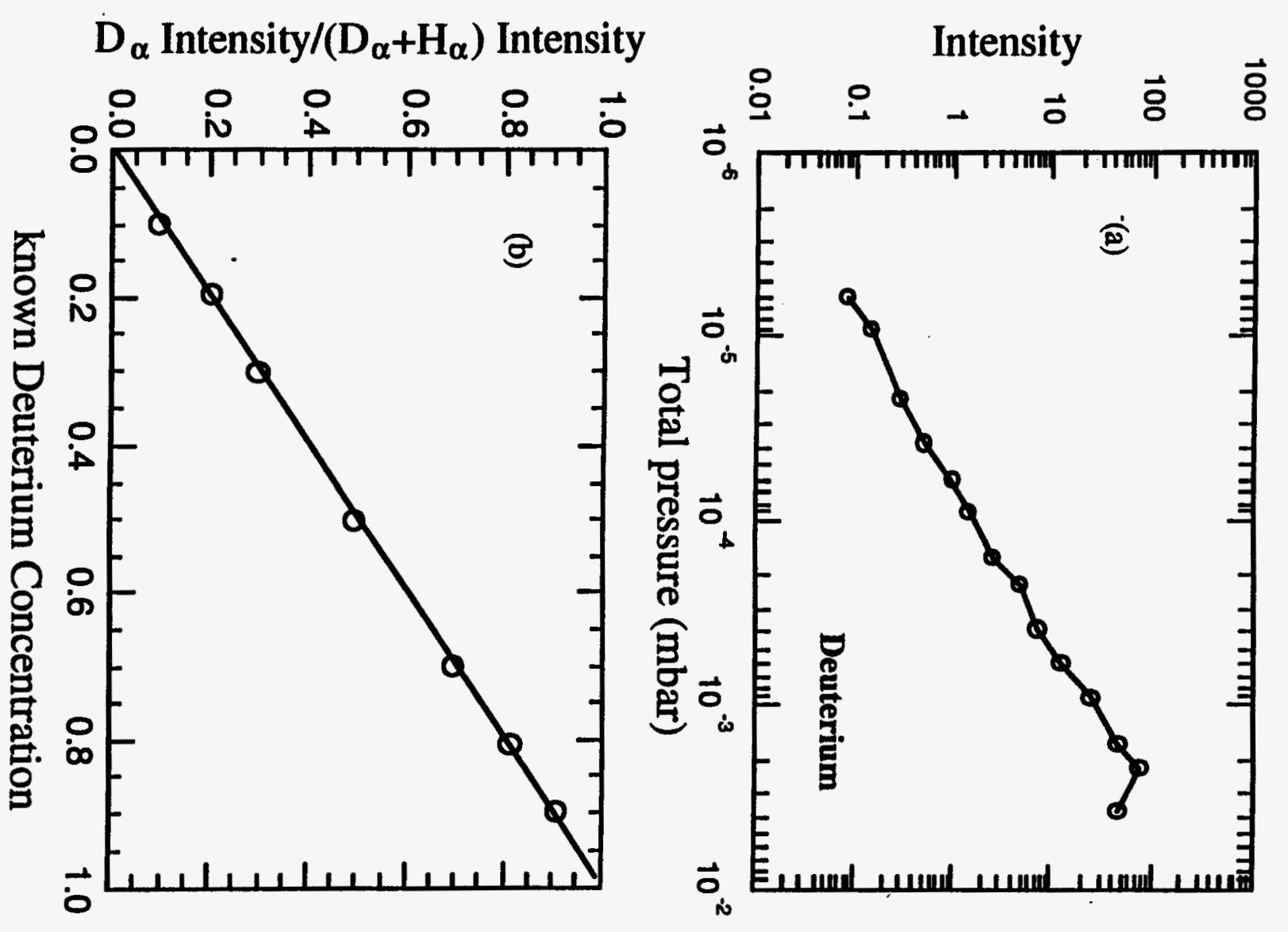




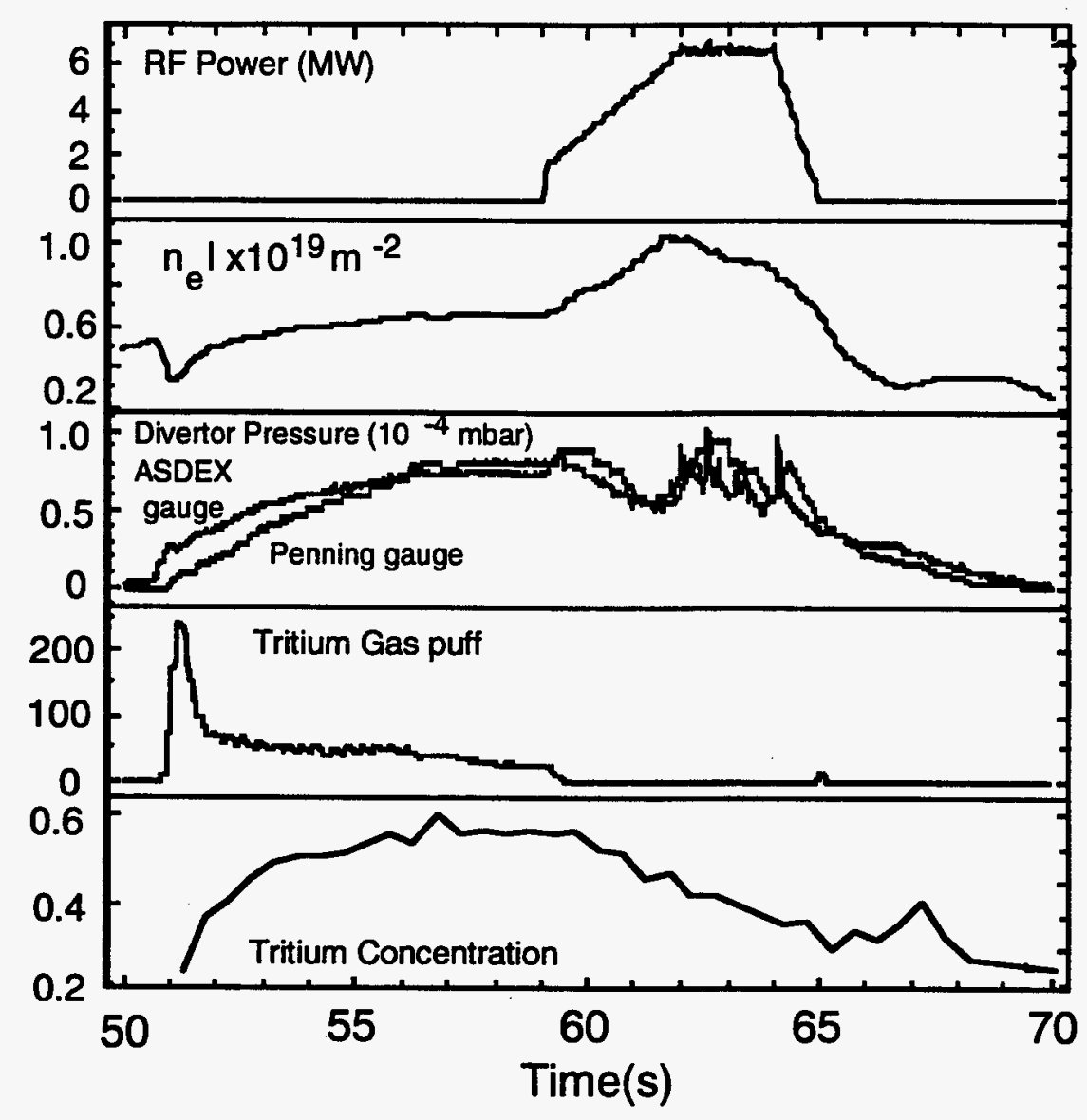

Fig. 4 


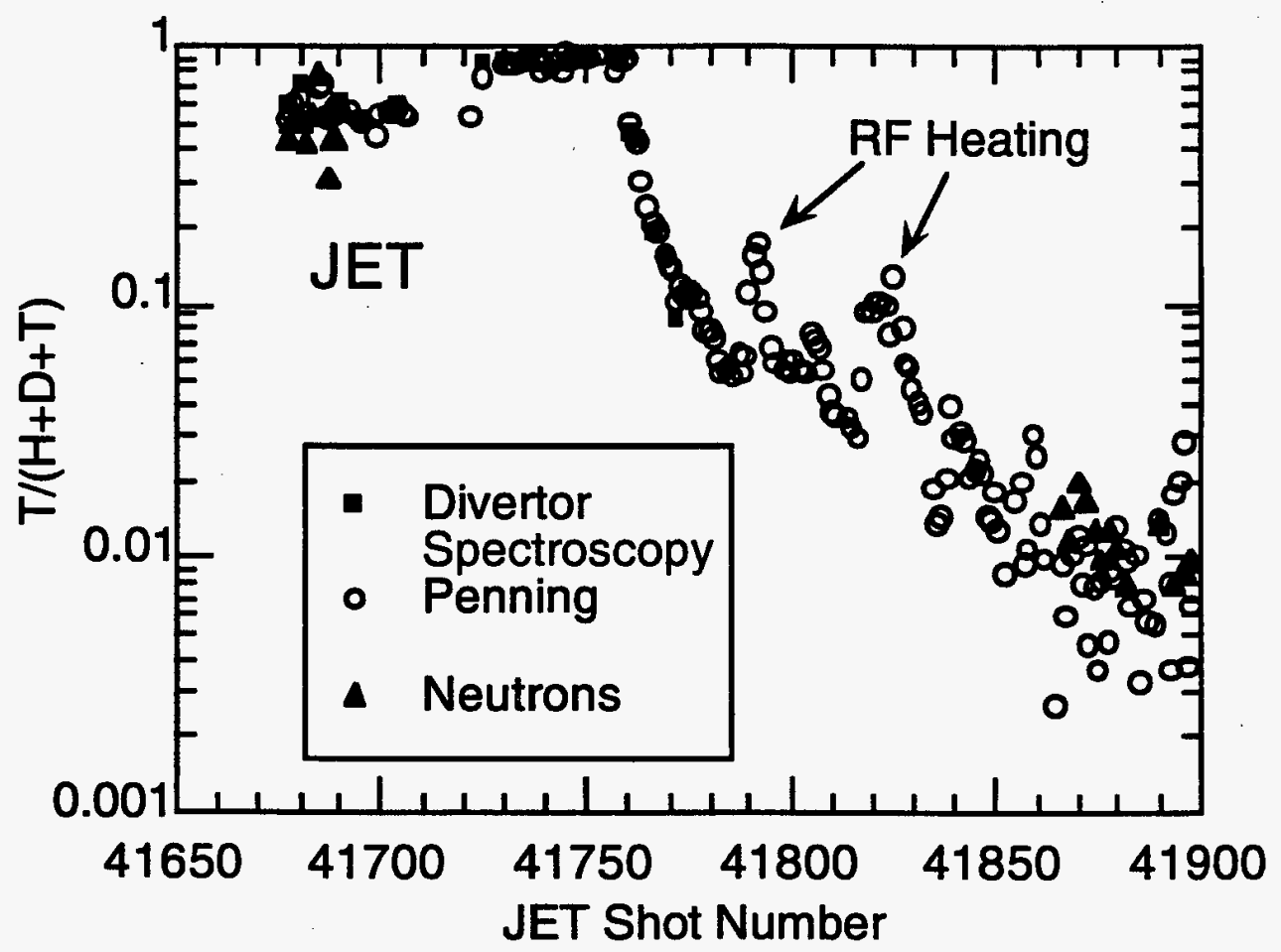

Fig. 5 


\section{M98003585}

|||||||||||||||||||||||||||||||||||||||||||||||||||||||||||

Report Number (14) OPNL/CP-.98498

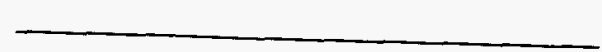

Publ. Date (11)

Sponsor Code (18) $\mathrm{DOR}, \mathrm{XF}$
UC Category (19) UC-126, DOE/ER

\section{6}

DTIC QUATITY INTPECTTHD 1 